\title{
Study Based on MMSE Channel Estimation for IEEE 802.15.3c PHY Realization Method
}

\author{
Linqing $\mathrm{Qi}^{\mathrm{a}}$, Rufei Xia ${ }^{\mathrm{b}}$ \\ South China University Of Technology Center of network, Guangzhou, China \\ aemail:676365779@qq.com; bemail:1499321258@qq.com
}

Keywords: IEEE 802.15.3c, HSI PHY, MMSE, OFDM

\begin{abstract}
IEEE 802.15.3c standard is the first IEEE wireless standard for data rates over $1 \mathrm{~Gb} / \mathrm{s}$ in $60 \mathrm{GHz}$ band. The dissertation create a simulation system, which adopt the HSI PHY of IEEE 802.15.3c and OFDM modulation method, realize the full process from the transmitter to the receiver. By using three types of channel, the dissertation analyses the performance of the standard under different channel conditions. The performance include the error rate in high speed wireless transmission and the feasibility of realizing Gigabit transmission. At last, by using the MMSE channel estimation algorithm, the system performance is improved, and the accuracy of data transmission is improved.
\end{abstract}

\section{Introduction}

IEEE 802.15.3c standard is located in the frequency band of $60 \mathrm{GHz}$, which belongs to millimeter wave communication[1-2]. It is a short distance high speed wireless connection technology. The $60 \mathrm{GHz}$ millimeter wave communication system mainly includes MAC and PHY. The high rate transmission is guaranteed by the PHY, which mainly defines the signal transceiver (front of RF and end of baseband processing), antenna control and modulation methods, etc[3].802.15.3c working group put forward three different physical layer mode: (SC PHY ) single carrier physical layer, (HSI PHY) high speed interface physical layer, (AV PHY) video physical layer. In this paper, HSI PHY based on OFDM is used, data transmission rate is $1.54-5.78 \mathrm{~Gb} / \mathrm{s}$, block size is 512 .As a result, low density parity check (LDPC), 16-QAM modulation is the only choice[4].

Data communication in wireless environment is often limited by the influence of noise and inter symbol interference (ISI).Especially high speed data transmission, the system error rate will be increased significantly. The channel estimation can improve the accuracy of the received data and improve the performance of the communication system. In this paper, the minimum mean square error (MMSE) channel estimation is used to compare the bit error rate (BER) results under different communication environments, and the performance of the IEEE 802.15.3c standard in the corresponding communication environment is obtained.

\section{MMSE Channel Estimation Algorithm}

Channel estimation is the key technique of channel equalization. In this paper, the simulation system environment is fast fading channel, using MMSE method based on comb pilot estimation for channel estimation, and assisted by LS algorithm for comparison.

The MMSE minimizes the mean square error by using the two order statistics of the channel state. Simply, by seeking a suitable channel impulse response (CIR), which makes the difference between the received data calculated by the CIR and the actual data the smallest.[5]

$$
H_{\text {mmse }}=R_{\mathrm{HH}}\left(R_{\mathrm{HH}}+\sigma_{n}^{2}\left(X X^{\mathrm{H}}\right)^{-1}\right)^{-1} H_{1 \mathrm{~s}}
$$

Whenever the signal $X$ changes, the matrix $R_{\mathrm{HH}}$ to be changed, and the inverse calculation is 
complex. In order to simplify the MMSE algorithm, replacing the $\left(X X^{\mathrm{H}}\right)$ with $\mathrm{E}\left\{\left(X X^{\mathrm{H}}\right)^{-1}\right\}$. The performance degradation caused by this approximation can be ignored.

\section{Description Of IEEE802.15.3c Physical Layer Model}

Baseband Model Of OFDM System. The simulation model of 802.15.3c IEEE physical layer is proposed in this paper based on OFDM (orthogonal frequency division multiplexing). The baseband model of OFDM system is shown in Figure 1.

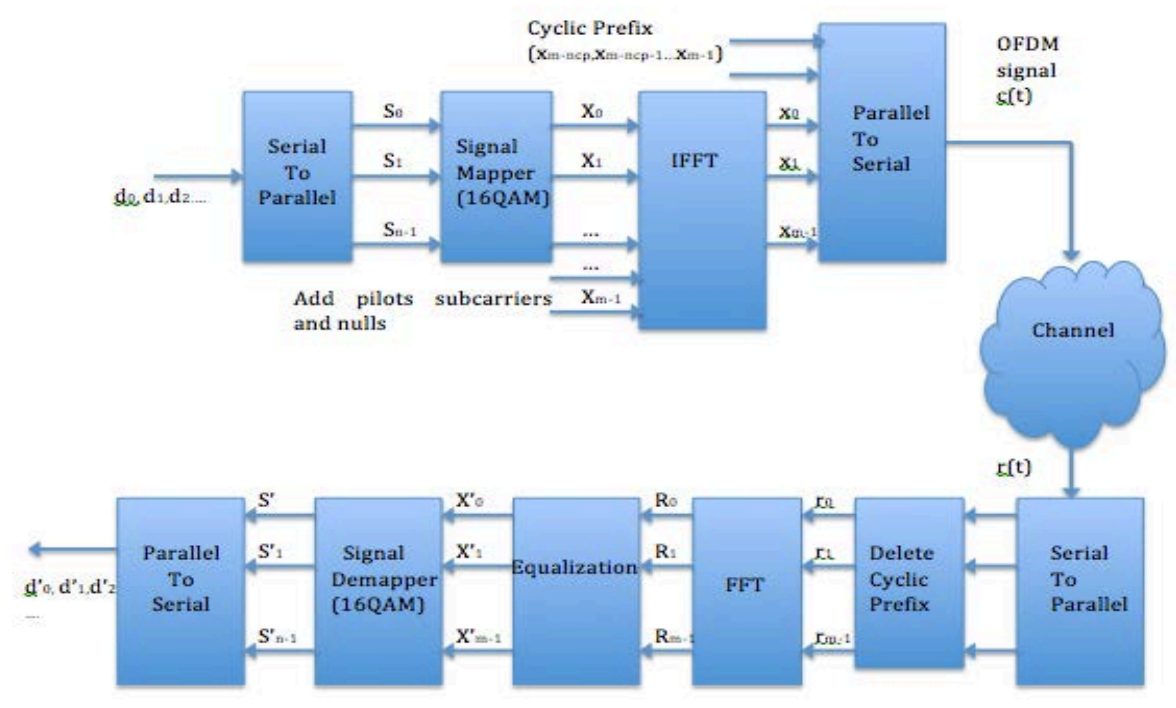

Fig.1.The baseband model of OFDM system

OFDM Modulation. Each OFDM symbol sent by transmitter contains 576 subcarriers, which contains 336 data subcarriers, 16 pilot subcarriers, 16 protection sub carrier spacing, 141 null subcarriers, 3 DC subcarrier and 64 cyclic prefix. 336 data sub carrier is generated by Matlab. Transform bit stream into complex by 16QAM modulation, 16QAM constellation mapping is shown in Figure 2.

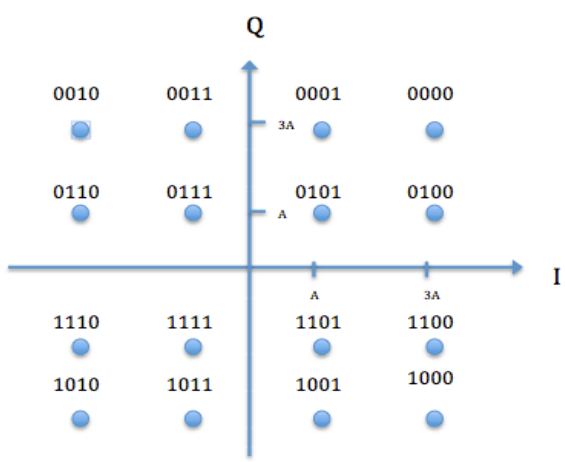

Fig.2.16QAM constellation

$I$ and $Q$ stand for "In-phase" and "Quadrature" components, the symbol generated with 16QAM is [5]:

$$
X(t)=I(t) \cos \left(2 \pi f_{0} t\right)+Q(t) \sin \left(2 \pi f_{0} t\right)
$$

Where: $X(t)$ is the signal mapped in the constellation, $f_{0}$ is the carrier frequency.

Add the pilot to improve the accuracy of data, add zero added between OFDM symbols to protect the OFDM from interference between symbols. The positions of 16 pilot subcarriers are: $[-166,-144,-122,-100,-78,-56,-34,-12,12,34,56,78,100,122,144,166]$. The positions of 141 guard subcarriers and 16 guard subcarriers are [-256: -178 178:255]. The positions of 3 DC subcarriers are $[-1,0,1]$.

Channel. Indoor wireless channel model is simulated by a FIR (impulse response finite) filter and Gauss white noise. The function is 


$$
y_{t}=x_{t} \times h_{t}+n_{t}
$$

Impulse response of the channel is

$$
h_{t}=\delta_{t}
$$

This channel does not have any ISI and ICI, the performance of BER is mainly decided by noise, we can get the theoretical value of BER by formula calculation.

$$
B E R_{M Q A M}=\frac{3}{2 k} \operatorname{erfc}\left(\sqrt{\frac{k E b}{10 N_{0}}}\right)
$$

Where: $E_{b}$ is average energy of a bit, $\mathrm{N}_{0}$ is Gauss white noise spectral density, $k=\log _{2}(M)$.

The erfc equation is defined as:

$$
\operatorname{erfc}(x)=\frac{2}{\sqrt{\pi}} \int_{x}^{\infty} e^{-\frac{y^{2}}{2}} d y
$$

So, we can get a conclusion to 16QAM $(M=16)$ as follow:

$$
B E R_{16 Q A M}=\frac{3}{8} \operatorname{erfc}\left(\sqrt{\frac{4 E b}{10 N_{0}}}\right)
$$

Its transfer function is

$$
H_{f}=1
$$

Impulse response of the channel is

$$
h_{t}=\delta_{t}+\frac{1}{2} \delta_{t-1}+\frac{1}{4} \delta_{t-2}
$$

Its transfer function is

$$
H_{f}=1+\frac{1}{2} e^{-2 j \pi f}+\frac{1}{4} e^{-4 j \pi f}
$$
$h$ is

Impulse response of channel is based on the size of the environment. Channel impulse response

$$
\begin{array}{r}
h_{\tau}=\sum_{k=0}^{N} b_{k} \delta_{\tau-k} \\
b_{k}=\sqrt{P_{k}} e^{j \varphi}, \quad \varphi \in[0,2 \pi]
\end{array}
$$

Before simulation, the following parameters are needed. Power delay distribution is

$$
P_{k}=e^{-\frac{k T_{s}}{\tau}}, \quad \text { for } k \geq 1
$$

Time delay is

$$
\tau \approx \frac{a}{c}
$$

Where $a$ is the size of environment, $c$ is the velocity of light, $\tau$ is the time delay, $T_{s}$ is the sampling time, $P_{k}$ is the power profile, $h_{\tau}$ is the channel impulse response. The model is applicable to the complex environment with dense multichannel. This paper refers to another article [4], but only one set of channel parameters in that paper is used, which is a set of Channel impulse response parameters [0.87 0.1 0.030 .010 .0050 .001 ]

OFDM Demodulation.1)The receiver divides the received signal into a number of sub carriers. And a OFDM symbol contains 576 sub carriers, 64 of which are cyclic prefix.

The delay in receiver is only a phase rotation for each sub carrier. This rotation will not generate ICI in the demodulation process. So the cyclic prefix has completed its task now and it should be removed before the demodulation. 
FFT transform, transforming the signal from the time domain to the frequency domain.

$$
X_{m, k}=\sum_{n=0}^{N-1} e^{-j \frac{2 \pi}{N} n k} x_{m, n}
$$

Where: $m$ is the $m_{\text {th }}$ OFDM symbol, $k$ is the $k_{\text {th }}$ subcarrier of the $m_{\text {th }}$ OFDM symbol after FFT, $n$ is the $n_{\text {th }}$ subcarrier of the $m_{\text {th }}$ OFDM symbol before FFT, $N$ is the number of the subcarriers for a symbol.

Channel equalization techniques is used to improve the accuracy of received signals. Channel equalization includes channel estimation which based on MMSE algorithm, linear interpolation and zero forcing equalization. In the linear interpolation algorithms, assuming that the two similar pilot is $\left(x_{0}, h_{0}\right)$ and $\left(x_{1}, h_{1}\right) \cdot(x, H)$ is the estimated point, then the interpolation equation is

$$
h=\frac{h_{1}-h_{0}}{x_{1}-x_{0}} \times\left(x_{1}-x_{0}\right)+h_{0}
$$

In this system, two comb pilot points need to be inserted in more than 20 points, the value of the insertion point is the Estimated value of channel frequency response without Pilot points.

\section{Simulation Results And Analysis Under Different Channel Models}

Gauss White Noise Channel. The system is simulated on the Gauss white noise channel in 0-10dB range. The theoretical value, the simulation value of the known channel parameters, the simulation value based on the LS estimation and the simulation value based on the MMSE estimation are compared. As shown in Figure 3(a):The simulation based on the MMSE channel estimation is significantly better than the simulation based on the LS channel estimation. In the case of the same bit error rate, SNR of the former is smaller than the latter about $0.5 \mathrm{~dB}$.

Gauss White Noise Channel With Multipath Interference. The system is simulated on the Gauss white noise and impulse response interference channel in 0-10dB range. The channel impulse response (CIR) is $[1,1 / 2,1 / 4]$. The theoretical value, the simulation value of the known channel parameters, the simulation value based on the LS estimation and the simulation value based on the MMSE estimation are compared. As shown in Figure 3(b): The simulation based on the MMSE channel estimation is between the simulation based on the LS channel estimation and the simulation based on known channel parameters. The difference among the three is small. In the case of the same bit error rate, SNR of the MMSE is smaller than the LS about $0.5 \mathrm{~dB}$.

Wireless Channel For Real Indoor Environment. The system is simulated on the real indoor environment channel in $0-10 \mathrm{~dB}$ range. The theoretical value, the simulation value of the known channel parameters, the simulation value based on the LS estimation and the simulation value based on the MMSE estimation are compared. As shown in Figure 3(c):In the case of the same bit error rate, SNR of the MMSE is also smaller than the LS about $0.5 \mathrm{~dB}$.

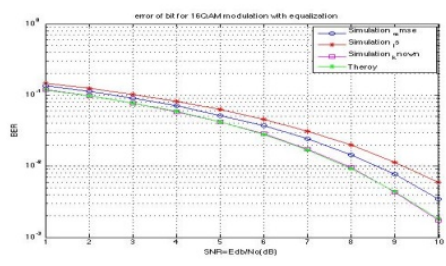

(a)Gauss channel (b)Gauss with multipath interference channel (c)real indoor channel

Fig.3.BER of different channel in simulation

\section{Summary}

802.15.3c IEEE standard has millimeter wave characteristics, so it is not suitable for long distance transmission, but it has superior performance in the indoor short distance transmission. In this paper, the simulation results of LS estimation and the simulation results of MMSE estimation are compared under three different kinds of channel. The result of MMSE algorithm is more accurate 
and more close to the real parameters of the channel than the result of LS algorithm. In the case of the same bit error rate, SNR of the former is smaller than the latter about $0.5 \mathrm{~dB}$. In certain signal to noise ratio, the physical layer of IEEE 802.15.3c standard has good performance. It can achieve G bit per second transmission rate with an acceptable bit error rate (BER).

\section{Reference}

[1] T. Baykas, Chin-Sean Sum, Zhou Lan, Junyi Wang, M.A. Rahman, H. Harada, S.K. Kato, ”IEEE 802.15.3c:The First IEEE Wireless Standard for Data Rates over $1 \mathrm{~Gb} / \mathrm{s}$," Communications Magazine, IEEE, vol.49, pp.114-121, July 2011.

[2] Hao Xu, Lucent Technol. Bell Labs. Spatial and temporal characteristics of 60-GHz indoor channels[J]. IEEE Journal on Selected Areas in Communications. Apr 2002. vol. 20,Issue:3: pp. 620-630

[3] Rui Sun. Technology and research prospects of millimeter wave wireless communication system [N]. Science of Telecom. 2007 twelfth phase

[4] M. Liso Nicolas, M. Jacob, T. Kurner. Physical layer simulation results for IEEE 802.15.3c with different channel models[J]. Advances in Radio Science. 2011.vol.9, pp.173-177

[5] Sandell M., van de Beek, J.-J. OFDM channel estimation by singular value decomposition[J]. Vehicular Technology Conference.1996, 'Mobile Technology for the Human Race., IEEE 46th. 28 Apr-1 May 1996.vol.2 :pp.923-927

[6] Jidong Zhang, Baoyu Zheng, channel estimation and research progress of OFDM based on pilot [J]. Journal of communication based on the issue of 24.No.112003. 